\title{
Tool Wear Prediction Based on Multidomain Feature Fusion by Attention-Based Depth-Wise Separable Convolutional Neural Network in Manufacturing
}

\section{Guofa Li}

Jilin University

Yanbo Wang ( $\nabla$ wangyb18@mails.jlu.edu.cn )

Jilin University

Jialong He

Jilin University

Yongchao Huo

Jilin University

\section{Research Article}

Keywords: Tool wear prediction, Depth-wise separable convolutional neural network, Feature fusion, Position encoding, Self-attention mechanism, Manufacturing.

Posted Date: July 12th, 2021

DOl: https://doi.org/10.21203/rs.3.rs-681400/v1

License: (9) This work is licensed under a Creative Commons Attribution 4.0 International License. Read Full License 


\title{
Tool wear prediction based on multidomain feature fusion by attention-based depth-wise separable convolutional neural network in manufacturing
}

\author{
Guofa $\mathrm{Li}^{1,2} \cdot$ Yanbo Wang ${ }^{1,2} \cdot$ Jialong $\mathrm{He}^{1,2} \cdot$ Yongchao Huo $^{1,21}$
}

\begin{abstract}
Tool wear during machining has a great influence on the quality of machined surface and dimensional accuracy. Tool wear monitoring is extremely important to improve machining efficiency and workpiece quality. Multidomain features (time domain, frequency domain and time-frequency domain) can accurately characterise the degree of tool wear. However, manual feature fusion is time consuming and prevents the improvement of monitoring accuracy. A new tool wear prediction method based on multidomain feature fusion by attention-based depth-wise separable convolutional neural network is proposed to solve these problems. In this method, multidomain features of cutting force and vibration signals are extracted and recombined into feature tensors. The proposed hypercomplex position encoding and high dimensional self-attention mechanism are used to calculate the new representation of input feature tensor, which emphasizes the tool wear sensitive information and suppresses large area background noise. The designed depth-wise separable convolutional neural network is used to adaptively extract high-level features that can characterize tool wear from the new representation, and the tool wear is predicted automatically. The proposed method is verified on three sets of tool run-to-failure data sets of three-flute ball nose cemented carbide tool in machining centre. Experimental results show that the prediction accuracy of the proposed method is remarkably higher than other state-of-art methods. Therefore, the proposed tool wear prediction method is beneficial to improve the prediction accuracy and provide effective guidance for decision making in processing.
\end{abstract}

Keywords Tool wear prediction, Depth-wise separable convolutional neural network, Feature fusion, Position encoding, Selfattention mechanism, Manufacturing.

\section{Introduction}

In the cutting process, the slight wear or damage of cutting tool reduces the quality of processing parts. The serious damage of cutting tool leads to the scrapping of parts, interruption of cutting process, and machine tool damage. In the actual machining process, approximately $20 \%$ of downtime is caused by tool wear $[1,2]$. Therefore, real-time online monitoring of tool wear is of great importance to improve product quality and production efficiency. To avoid the interruption of machining process, researchers usually collect vibration, cutting force and acoustic emission (AE) signals for tool wear monitoring.

Existing research shows that time domain, frequency domain and time-frequency domain features can well characterise the degree of tool wear. Morgan et al. [3] extracted time domain features (e.g. mean and maximum) and frequency domain features (e.g. wavelet entropy) from cutting force and vibration signals to characterise tool wear. Zhang et al. [4] extracted four energy features on the basis of empirical mode decomposition and eight energy features on the basis of time-frequency spectrum from $\mathrm{AE}$ signals for tool wear monitoring. Wang et al. [5] combined the frequency domain, time domain and time-frequency domain features of force and vibration signals (e.g., variance, maximum wavelet energy) for tool wear monitoring. However, these extracted features only aim at specific signals or domains and cannot realise a universal characterisation of tool wear.

As the mainstream method in data-driven tool wear monitoring, machine learning has been widely used.
Pandiyan et al. [6] combined genetic algorithm and support vector machine (SVM) for belt wear monitoring in grinding process. Genetic algorithm was used to extract a small number of tool wear sensitive features from the set of time domain and frequency domain features. Kong et al. [7] used an integrated radial basis function-based kernel principal component analysis (KPCA) to fuse the multidomain features extracted from the original signals. Gaussian regression was applied to predict the tool wear value and the corresponding confidence interval in real time. Wang et al. [5] used KPCA to fuse frequency domain, statistical domain and time-frequency domain features and utilised support vector regression (SVR) to predict tool wear. $\mathrm{Li}$ et al. [8] used gradient lifting decision tree to select the optimal feature subset from the set of multidomain features. They applied a hybrid classification restricted Boltzmann machine to identify the tool wear state. $\mathrm{Wu}$ et al. [9] extracted multidomain features from vibration and cutting force signals and selected the optimal features in terms of Pearson correlation coefficient, monotonicity and autocorrelation. An adaptive network fuzzy inference system was used to fuse the selected features. However, researchers have adopted some feature dimension reduction or feature selection methods to reduce the number of multidomain features. This condition is due to the weak ability of the above shallow machine learning methods to learn highdimensional data and complex nonlinear relationships. The use of feature selection or feature dimension reduction methods takes time and effort and loses some tool wear sensitive information, thereby limiting the accuracy of tool wear prediction.

In recent years, deep learning has been widely used in

\footnotetext{
1 Guofa Li, 809808837@qq.com; $₫$ Yanbo Wang, wangyb18@mails.jlu.edu.cn; Jialong He, $1135464421 @$ qq.com; Yongchao Huo,yongchaohuo@foxmail.com

${ }^{1}$ Key Laboratory of CNC Equipment Reliability, Ministry of Education, Jilin University, Changchun, Jilin, China

${ }^{2}$ School of Mechanical and Aerospace Engineering, Jilin University, Changchun,130022, China
} 
tool wear monitoring due to its powerful data mining ability and the ability to overcome the above limitations. Fu et al. [10] designed a deep convolutional neural network (CNN) for tool wear monitoring in drilling process. The performance of the model was better than radial basis function SVM. Aghazadeh et al. [11] applied wavelet timefrequency transform to extract wavelet energy features and utilised CNN to predict tool wear in milling process. The prediction accuracy was higher than that of Bayesian ridge regression and SVR. Zhao et al. [12] used CNN to extract local features from the input signals. They utilised bidirectional long-term and short-term memory (LSTM) network to encode the dependence between features and applied multilayer perceptron (MLP) to predict tool wear. The proposed model was superior to linear regression, MLP and SVR. Cheng et al. [13] proposed a deep CNN model based on sound signal to recognise abrasive belt wear state. Chen et al. [14] combined a CNN and a bidirectional LSTM network with an attention mechanism for tool wear prediction in milling process. Huang et al. [15] proposed a new tool wear predicting method based on reshaped time series CNN. Its performance was better than SVR and SVR+KPCA. Martinez et al. [16] used Gramian angular difference field to visualise the vibration and force signal, and applied a CNN with Cifar-10 architecture to identify the tool wear state. Cao et al. [17] proposed a new method of tool wear state recognition based on spindle vibration signals. In this method, translation-invariant wavelet frames were used to obtain the time-frequency spectra of the signals. CNN was used to construct the relationship between the spectrum and the tool wear state. Song et al. [18] proposed a deep CNN with LeNet architecture based on spindle current signals for tool wear state recognition in milling process. Sun et al. [19] reconstructed the vibration, force and $\mathrm{AE}$ signals into images and sent them to the designed residual CNN for tool wear prediction. Li et al. [20] proposed a new residual dense neural network based on vibration signals for online tool wear monitoring. Although the performance of these deep learning methods is better than that of traditional methods, they still can be improved. The extracted features of these methods often come from specific signals or domains and cannot form a generic solution for any signal or domain. The feature sets used in these methods have low dimension and small capacity, hindering the further improvement of prediction accuracy.

As a variant of standard convolution, depth-wise separable convolution has achieved remarkable success in image processing, natural language processing, machine fault diagnosis and other fields [21]. Shang et al. [21] proposed a densely connected and depth-wise separable CNN to classify polarimetric synthetic aperture radar images. Compared with the standard CNNs, the classification accuracy was improved by $10.2 \%$. Huang et al. [22] proposed a method of rolling bearing remaining useful life prediction based on transfer depth-wise separable CNN. Experimental result showed that the proposed method can improve the prediction accuracy and robustness. Chollet et al. [23] proposed a new depth-wise separable CNN model. The performance of the proposed model on ImageNet data sets was better than that of Inception V3. Xin et al. [24] proposed a depth-wise separable CNN for fault diagnosis of the attachment of marine current turbine. Compared with the standard CNN, the depth-wise separable CNN has the advantages of less trainable parameters and low computational complexity, which helps to further increase the number of convolution layers, and further improve the performance of the model [25]. Although depth-wise separable convolution has achieved remarkable success in image classification and other fields, it has not been applied in the field of tool wear prediction.

Convolutional and pooling operation of depth-wise separable CNN are building build blocks that deal with one local neighbourhood at a time, which can suppress local background edge and impulse noise, but it is difficult to suppress large area of slowly changing background noise [26-28]. Since attention mechanism can emphasize useful information and suppress background noise from the perspective of the whole input tensor, existing researches often embed attention mechanism into CNN. Zeng et al. [29] proposed a convolutional neural network with self-attention mechanism for tool wear monitoring. Wang et al. [30] proposed a new multiscale $\mathrm{CNN}$ with attention mechanism to predict the remaining useful life of machinery. Huang et al. [31] proposed a self-attention-based CNN for document classification, which models documents from two levels: words and sentences and sentences and documents. Compared with the existing methods, the proposed method had the highest accuracy. The self-attention mechanism can't capture the position information between different elements in the input tensor, so it is often combined with the position encoding algorithm to enhance its performance. Vaswani et al. [32] combined sinusoidal position encoding and selfattention mechanism to solve machine translation task, and achieved higher single-model bilingual evaluation understudy score. Liu et al. [33] proposed a neural network model for tool wear prediction based on sinusoidal position encoding and self-attention mechanism. Shaw et al. [34] combined relative position encoding and self-attention mechanism for machine translation task. Considering that the transformer models were easily limited by fixed length context, Dai et al. [35] proposed a new transformer-XL model for natural language modelling task, which combined segmented relative position encoding and self-attention mechanism. Although these neural network models with attention mechanism have achieved good performance, they still can be improved. On the one hand, the existing attention mechanisms use sinusoidal position encoding and other position encoding algorithms to encode the position information. These position encoding algorithms can't model the order relationship (i.e., adjacency and precedence) between different elements in the input tensor. On the other hand, the existing attention mechanisms can only deal with one-dimensional input, but cannot deal with highdimensional input, which hinders the further improvement of its ability to suppress large area of slowly changing background noise.

A new tool wear prediction method based on multidomain feature fusion by attention-based depth-wise 
separable convolutional neural network (ADSCNN) is proposed to improve the prediction efficiency and accuracy. Firstly, multidomain features are extracted from multisensory signals. Then, the proposed hypercomplex position encoding and high dimensional self-attention mechanism are used to calculate the new representation of input feature tensor, which emphasizes the tool wear sensitive information and suppresses large area background noise. Finally, the proposed depth-wise separable CNN is used to model the nonlinear relationship between tool wear and the new representation. The rest of this paper is arranged as follows: Section 2 briefly introduces the related theories and elaborates the proposed theoretical methods. Section 3 introduces the framework and architecture of the proposed tool wear prediction method. Section 4 presents the experimental data sets. Section 5 analyses, compares and discusses the experimental results. Section 6 summarises the content of this paper.

\section{Related theories}

\subsection{Hypercomplex position encoding}

Since CNNs are easily disturbed by noise in input tensor, it is necessary to use the proposed high dimensional selfattention mechanism to enhance useful information in input tensor and suppress useless information. The proposed high dimensional self-attention mechanism relies on proposed hypercomplex position encoding to encode the global absolute position information of each feature vector and their inner sequential and adjacent relationships.

Suppose that a feature matrix $F M$ contains $n$ feature vector of $D$ dimension, which are expressed as $f m_{1}, \ldots f f m_{n}$ respectively. The hypercomplex position encoding is expressed as Eq. 1.

$$
f\left(f m_{p o s}, p o s\right)=f m_{p o s} \text { e } g(p o s), g(p o s) \in i^{D}
$$

where $\odot$ denotes elementwise multiplication. $f m_{p o s}$ represents the feature vector at position index pos in the feature matrix. The function $g(\bullet)$ defines a mapping $g$ : $N \rightarrow C^{D}$ from a discrete position index pos to $D$-dimensional vector. The function $g(\bullet)$ is a continuous function that changes smoothly with the change in position index pos. Therefore, the position encoding representations of different positions are interrelated. Position index pos is a positive integer and represents the position of each feature vector in the feature matrix. The function $g(\bullet)$ needs to satisfy properties A and B.

Property A: As shown in Eq. 2, the function $g(\bullet)$ should be a position-independent offset transformation.

$$
\exists z \in £, \forall p o s \in ¥, g(\text { pos }+n)=g(\text { pos }) * z^{n}
$$

Property A ensures that the transformation from one position to its adjacent position is a smooth transformation.

Property B: As shown in Eq. 3, the function $g(\bullet)$ should be bounded.

$$
\exists \delta \in \mathfrak{i}^{+}, \forall p o s \in ¥,|g(p o s)| \leq \delta
$$

Property B ensures that the function $g(\bullet)$ can deal with large position index pos.

The function $g(\bullet)$ is expressed as Eq. 4-5. The function $g(\bullet)$ satisfies properties A and B.

$$
\begin{aligned}
& g(p o s)=[g(\operatorname{pos}, 1), \ldots ., g(\operatorname{pos}, D)] \\
& g(p o s, d)=\exp \left(i\left(w_{d}^{i} * \operatorname{pos}+\theta_{d}^{i}\right)\right) \\
& * \exp \left(j\left(w_{d}^{j} * \operatorname{pos}+\theta_{d}^{j}\right)+z\left(w_{d}^{k} * p o s+\theta_{d}^{k}\right)\right)
\end{aligned}
$$

The function $g(\bullet)$ encodes each embedded dimension $d$ of each position index pos as a quaternion. The quaternion consists of a real part and three imaginary parts, which form a $4 \mathrm{D}$ vector. Hypercomplex position encoding encodes the input feature matrix with a size of $\mathrm{N} \times D$ into the output feature tensor with a size of $N \times D \times 4$.

In the training process of the proposed ADSCNN model, the parameters $\left(w_{d}^{i}, \theta_{d}^{i}, w_{d}^{j}, \theta_{d}^{j}, w_{d}^{k}\right.$ and $\left.\theta_{d}^{k}\right)$ are updated by using an error backpropagation algorithm.

\subsection{High dimensional self-attention mechanism}

After hypercomplex position encoding, high dimensional self-attention mechanism is used to obtains a new representation of the input feature tensor. The high dimensional self-attention mechanism is expressed as Eq. 6.

$$
\begin{aligned}
& \text { Attention=Attention }\left(V W_{Q}, V W_{K}, V W_{V}\right) W_{C} \\
& \text { Attention }\left(V W_{Q}, V W_{K}, V W_{V}\right)=\left[\begin{array}{l}
\sum_{j} \alpha_{1, j} v_{j} W_{V} \\
\ldots \\
\sum_{j} \alpha_{N, j} v_{j} W_{V}
\end{array}\right] \\
& \alpha_{i, j}=\operatorname{soft} \max \left(e_{i j} / \sum_{j=1}^{n} e_{i j}\right) \\
& e_{i j}=\frac{\left\langle v_{i} W_{Q}, v_{j} W_{K}\right\rangle}{\left\|v_{i} W_{Q}\right\|_{F} \bullet\left\|v_{j} W_{K}\right\|_{F}}=\frac{\operatorname{tr}\left(\left(v_{i} W_{Q}\right)^{T}\left(v_{j} W_{K}\right)\right)}{\left\|v_{i} W_{Q}\right\|_{F} \bullet\left\|v_{j} W_{K}\right\|_{F}}
\end{aligned}
$$

where $v_{i} W_{Q}, v_{j} W_{K}$ are matrices. $e_{i j}$ represents the similarity between matrix $v_{i} W_{Q}$ and $v_{j} W_{K} . W_{Q}, W_{K}, W_{V}$ and $W_{C}$ are learnable matrices. The function $\operatorname{tr}(\bullet)$ represents the sum of the main diagonal elements of a matrix. $\|\bullet\|_{F}$ represents the Frobenius-norm of a matrix. The value range of $e_{i j}$ is $[0,1]$. The closer $e_{i j}$ is to 1 , the higher the similarity between matrix $v_{i} W_{Q}$ and $v_{j} W_{K}$. The output Attention is a feature tensor with the same shape as input.

In the training process of the proposed ADSCNN model, the parameters $\left(W_{Q}, W_{K}, W_{V}\right.$ and $\left.W_{C}\right)$ are updated by using an error backpropagation algorithm.

\subsection{Depth-wise separable CNN}

Depth-wise separable CNNs have a strong ability of adaptive data mining and feature extraction and are widely used in fault diagnosis and prediction. A series of depth-wise separable convolution, standard convolution layers and pooling layers constitute the basic structure of depth-wise separable CNN. The depth-wise separable convolution is composed of depth-wise convolution layer and pointwise convolution layer.

The convolution layers can be regarded as the process of extracting local robust features from input feature map. The depth-wise convolution layer allocates a convolution kernel to each channel of the input feature map $X^{l-1}$, convolutes each channel with the corresponding convolution kernel, and generates the output feature map $X^{l}$ through the 
set activation function. The depth-wise convolution layer is expressed as Eq. 10.

$$
X_{m}^{l}=f\left(\sum_{i \in M_{m}} X_{i, m}^{l-1} * K_{m}+b_{m}^{l}\right)
$$

where $K$ represents the convolution kernel containing $M$ feature maps. $X_{m}^{l}$ represents the $m$-th feature map of the $l$ layer. $f(\bullet)$ represents the activation function. $M_{m}$ represents a set of inputs for calculating the $m$-th output. $b_{m}^{l}$ represents the $m$-th offset of the $l$-th layer. The input feature map $X^{l-1}$ contains $M$ channels. $X^{l}$ represents the output of depth-wise convolution layer. The $m$-th feature map of convolution kernel $K$ and the $m$-th channel of input feature map $X^{l-1}$ are convoluted.

In the training process of the proposed ADSCNN model, the parameters $(K$ and $b)$ are updated by using the error backpropagation algorithm.

$$
\begin{aligned}
& \frac{\partial \text { Loss }}{\partial K_{m}^{l}}=\sum_{p, q}\left(\left(\delta_{m}^{l}\right)_{p, q} *\left(P_{i}^{l-1}\right)_{p, q}\right) \\
& \frac{\partial \operatorname{Loss}}{\partial b_{m}^{l}}=\sum_{p, q}\left(\delta_{m}^{l}\right)_{p, q},
\end{aligned}
$$

where $\left(P_{i}^{l-1}\right)_{p, q}$ is the patch in $X_{i}^{l-1}$ that is multiplied elementwise by convolution kernel $K_{m}$ in the convolution process. Loss is the loss function. $\delta_{m}^{l}$ is the $m$-th element of the sensitivities in the $l$-th layer.

Pointwise convolution layer is a special case of the standard convolution layer. The pointwise convolution layer is mainly used to linearly combine different channels of the output feature map of the depth-wise convolution layer, and the size of its convolution kernel is $1 \times 1 \times M . M$ is the number of channels in the output feature map of the depth-wise convolution layer. The mathematical formula of standard convolution layer is suitable for pointwise convolution layer. The standard convolution layer is expressed as Eq. 12.

$$
X_{m}^{l}=f\left(\sum_{i \in M_{m}} X_{i}^{l-1} * K_{m}^{l}+b_{m}^{l}\right)
$$

where $K$ represents the convolution kernel. $f(\bullet)$ represents the activation function. $X^{l}$ represents the output of the standard convolution layer. $X_{m}^{l}$ represents the $m$-th feature map of the $l$ layer. $M_{m}$ represents a set of inputs for calculating the $m$-th output. $b_{m}^{l}$ represents the $m$-th offset of the $l$-th layer.

Similarly, the parameters of the standard convolution layer are updated by using the error backpropagation algorithm. The calculation formula for parameter update is expressed as Eq. 13.

$$
\begin{aligned}
& \frac{\partial \text { Loss }}{\partial K_{m}^{l}}=\sum_{p, q}\left(\left(\delta_{j}^{l}\right)_{p, q} *\left(P_{i}^{l-1}\right)_{p, q}\right) \\
& \frac{\partial \operatorname{Loss}}{\partial b_{m}^{l}}=\sum_{p, q}\left(\delta_{m}^{l}\right)_{p, q}
\end{aligned}
$$

where $\left(P_{i}^{l-1}\right)_{p, q}$ is the patch in $X_{i}^{l-1}$ that is multiplied elementwise by convolution kernel $K_{m}$ in the convolution process.

The pooling layer is mainly used to significantly reduce the spatial size of the input feature map and the number of parameters. The pool layer removes the redundant details, retains the information closely related to the task, and reduces the time and space complexities of the whole model. Commonly used pooling operations include average pooling, random value pooling and maximum pooling. The average pooling layer is expressed as Eq. 14.

$$
X_{m}^{l}=f\left(\beta_{m}^{l} * \operatorname{down}\left(X_{m}^{l-1}\right)+b_{m}^{l}\right)
$$

where $\operatorname{down}(\bullet)$ represents the down sampling function. $\beta_{m}^{l}$ is the multiplication bias, and $b_{m}^{l}$ is the addition bias. $f(\bullet)$ denotes the activation function.

Similarly, the parameters of pooling layer are updated by using the error backpropagation algorithm. The calculation formula for parameter update is expressed as Eq. 15.

$$
\begin{aligned}
& \frac{\partial \operatorname{Loss}}{\partial \beta_{m}^{l}}=\sum_{p, q}\left(\delta_{m}^{l} * \operatorname{down}\left(X_{m}^{l-1}\right)\right)_{p, q} \\
& \frac{\partial \operatorname{Loss}}{\partial b_{m}^{l}}=\sum_{p, q}\left(\delta_{m}^{l}\right)_{p, q},
\end{aligned}
$$

\section{Proposed methodology}

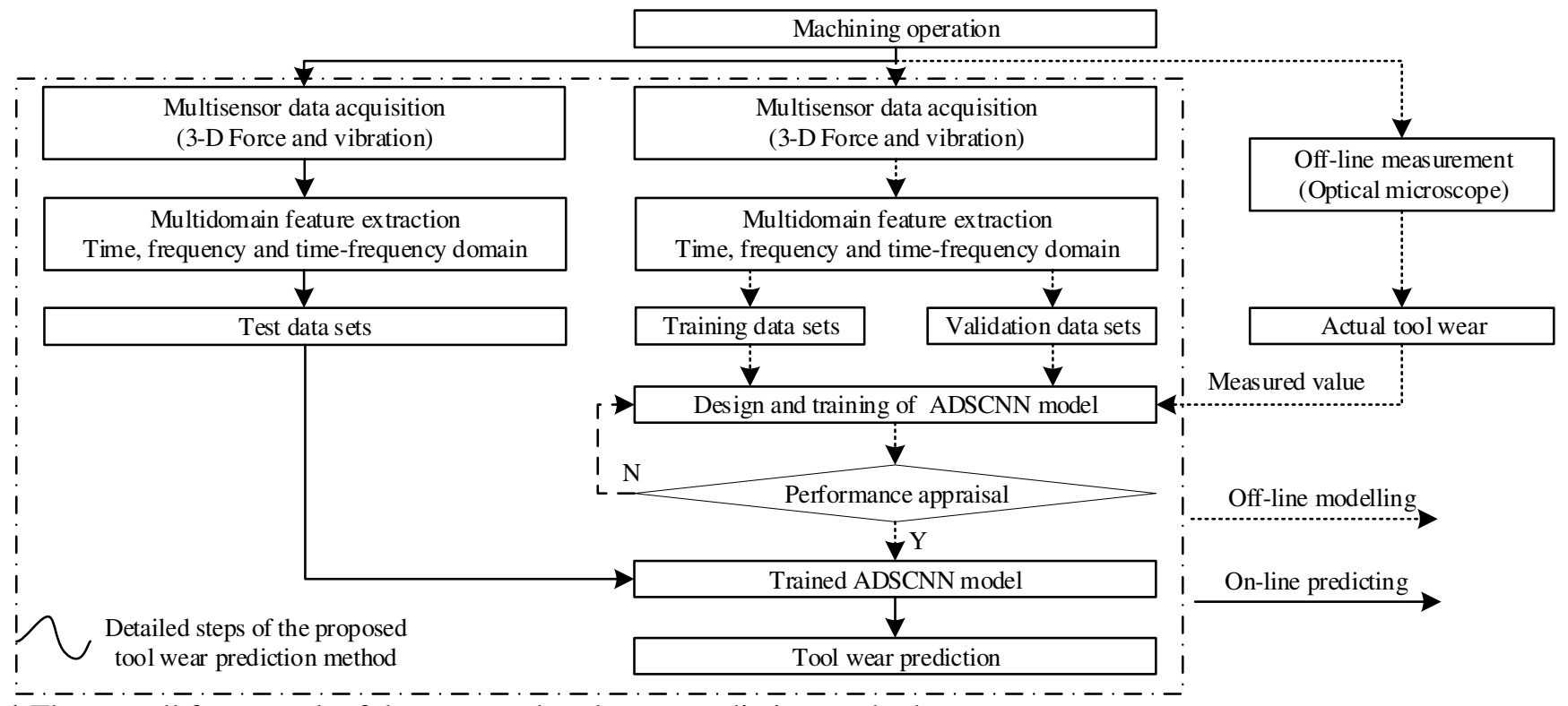

Fig. 1 The overall framework of the proposed tool wear prediction method 


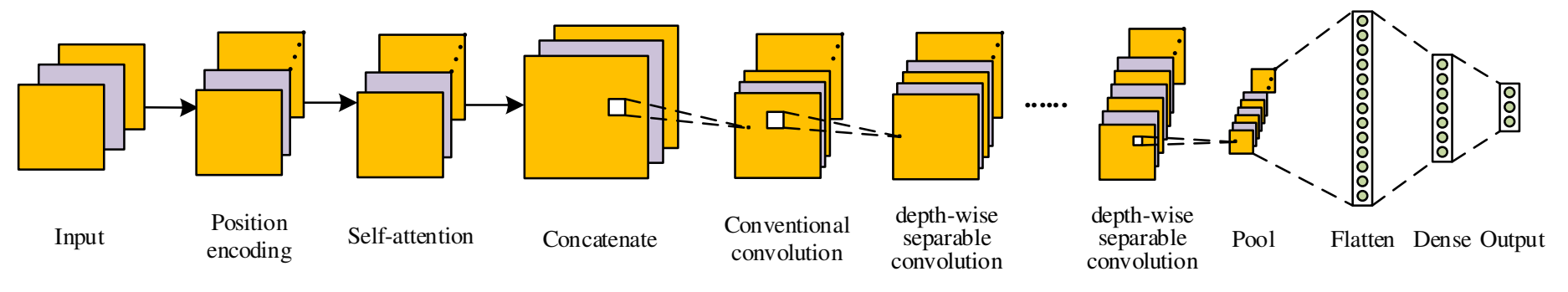

Fig. 2 The framework of proposed ADSCNN model

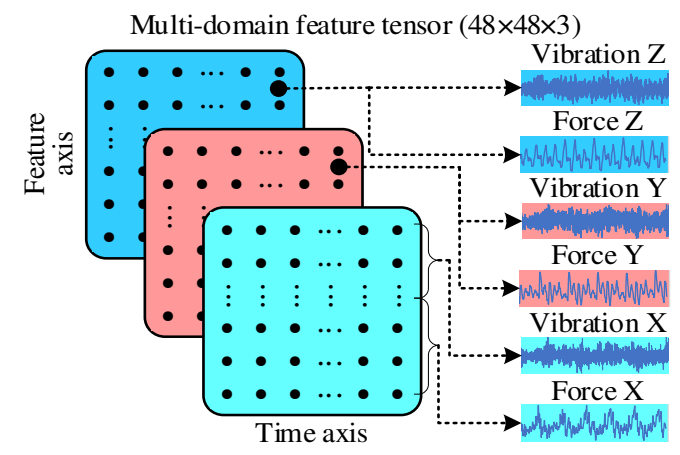

Fig. 3 The schematic diagram of input multidomain feature tensor

The framework of the proposed tool wear prediction method is shown in Fig. 1. In the offline modelling stage, multiple sensors are used to measure the cutting force and vibration signals in the milling process, and an optical microscope is used to measure the tool flank wear regularly. The multidomain features of sensor signals are extracted and normalised, and these features are recombined into multidomain feature tensors. The multidomain feature tensors and the corresponding tool wear value are taken as the sample data sets. The sample data sets are then divided into training and validation data sets. The training data sets is used to train the designed ADSCNN model, and the super parameters of ADSCNN model are adjusted on the basis of the validation data sets. After the training process, a usable ADSCNN model is obtained.

In the online prediction phase, multiple sensors are used to collect the cutting force and vibration signals in real time. Multidomain features are extracted and recombined into feature tensors as test data sets. The test data sets are input into the trained ADSCNN model for real-time tool wear prediction. The output value of the trained ADSCNN model needs inverse normalization to get the actual tool wear prediction value. Therefore, through off-line modelling and on-line prediction, the proposed method realizes adaptive fusion of multidomain features and tool wear prediction.

\subsection{Multisensory data acquisition}

In the milling process, the vibration signal is extremely sensitive to tool wear and contains abundant tool wear information. However, the vibration signal is vulnerable to the interference of sensor installation position and machining environment. Therefore, a vibration sensor is often used together with other sensors for tool wear monitoring. As an important tool wear monitoring signal, the cutting force gradually increases with the aggravation of tool wear. For vibration and cutting force signals, the sensitivity of different direction signals to tool wear is different.
Therefore, 3D cutting force and 3D vibration signals are collected by using vibration and cutting force sensors, and the tool flank wear is measured regularly by using an optical microscope.

\subsection{Multidomain feature extraction}

Many useless noises are found in the collected multisensory sensor signals. Thus, multidomain feature extraction is necessary. With the aggravation of tool wear, the time domain features of the signal inevitably change. The frequency spectrum and time-frequency spectrum of the signal also change. Specifically, the frequency domain and time-frequency domain features of the signal change. As shown in Table 1, 14 time domain features, 2 frequency domain features and 8 wavelet packet energy features are extracted from the vibration and cutting force signals in $\mathrm{x}, \mathrm{y}$ and $\mathrm{z}$ directions respectively. The original signal is divided into multiple signal segments to increase the sample size and the generalisation ability of the ADSCNN model. Each signal segment contains 1024 points. As shown in Fig. 3, each column of the x-direction multidomain feature matrix contains 48 features. The 48 features include 24 multidomain features extracted from $\mathrm{x}$-direction vibration signal samples and 24 multidomain features extracted from $\mathrm{x}$-direction cutting force signal samples. The features in the $\mathrm{y}$-direction and $\mathrm{z}$-direction multidomain feature matrices come from the vibration and cutting force signal samples in the y-direction and z-direction, respectively. Depth-wise separable CNN has achieved great achievements in image recognition, image segmentation and other fields. Its input is usually three-order square pixel tensor. Therefore, the time axis and feature axis of the multidomain feature matrices are set to the same dimension. For vibration and cutting force signals, the signals in three directions have certain correlation. For each sample, the multidomain feature matrices in $\mathrm{x}, \mathrm{y}$ and $\mathrm{z}$ directions are spliced into a three-order multidomain feature tensor, which is helpful for Depth-wise separable CNN to model the correlation between different directions. On the basis of the collected signals, multiple three-order multidomain feature tensors are extracted as the input of subsequent ADSCNN model.

\subsection{Design and training of ADSCNN model}

As the key of the proposed tool wear prediction method, ADSCNN model is mainly used to model the relationship between multidomain features and tool wear. The framework of the proposed ADSCNN model is shown in Fig. 2. Firstly, considering that the feature vectors at different times in each input matrix are interdependent, a new representation of each input feature matrix is calculated by using proposed 
hypercomplex position encoding and high dimensional selfattention mechanism, which emphasizes the tool wear sensitive information and suppresses large area background noise. Specifically, the input three-order feature tensor consists of three feature matrices. hypercomplex position encoding layer encodes each feature of each feature matrix into a $4 \mathrm{D}$ vector. Each input feature matrix with the size of $48 \times 48$ is encoded into feature tensors with a size of $48 \times 48 \times 4$. the input and output of high dimensional self-attention layer have the same size, so the size of the new representation of each input three-order feature tensors is $48 \times 48 \times 4$. The concatenate layer is used to splice the three three-order feature tensors into a larger three-order feature tensor with a size of $96 \times 96 \times 3$. Secondly, a standard convolution layer, six depth-wise separable convolutions and a pooling layer are used to extract high-level representation from the input feature tensor. Each depth-wise separable convolution consists of a depth-wise convolution layer and a pointwise convolution layer. Compared with standard convolution, depth-wise separable convolution significantly reduces the number of parameters and the complexity of computation, thereby helping to further increase the number of convolution layers. Superimposing multiple depth-wise separable convolutions is mainly used to extract high-level local robust features from the input feature tensor. The pooling layer is mainly used to compress features, reduce the size of the feature mapping plane and the amount of calculation and memory consumption. No gradient vanishing problem is found in the rectified linear unit function, and its convergence speed is fast in the training process. Thus, it is selected as the activation function of the convolution layer and pooling layer. Finally, a dense layer and an output layer are used to establish the nonlinear relationship between the input high-level representation and the normalised tool wear values. Table 2 shows the detailed parameters of each layer of the proposed ADSCNN model.

The ADSCNN model is trained by using the Frobenius norm of the difference between the predicted tool wear value $y^{p}$ and the actual tool wear value $y$. The loss function is expressed as Eq. 16.

$$
\text { Loss }=\frac{1}{2 N} \sum_{i=1}^{N}\left\|y_{i}^{p}-y_{i}\right\|
$$

where $N$ is the number of samples in the training data sets.

As a typical model training tool, $K$-fold cross validation is used to train the ADSCNN model for reducing the risk of overfitting and improving its generalisation ability. During the training process, the error backpropagation algorithm is used to update the super parameters for minimising the loss function.

\subsection{Performance appraisal of ADSCNN model}

To quantitatively and comprehensively evaluate the performance of the proposed ADSCNN model, this paper selects two classic evaluation indicators, namely, root mean squared error (RMSE) and mean absolute error (MAE). The smaller the values of MAE and RMSE, the closer the predicted value of flank wear width is to the actual value. The smaller the prediction error of the model, and the better the model performance. MAE and RMSE are expressed as Eq. 17 and 18, respectively.

$$
\begin{aligned}
& f_{\text {RMSE }}=\sqrt{\sum_{i=1}^{N}\left(y_{i}-y_{i}^{p}\right)^{2} / N} \\
& f_{M A E}=\sum_{i=1}^{N}\left|y_{i}-y_{i}^{p}\right| / N
\end{aligned}
$$

where $N$ represents the number of samples in the test data sets, $y_{i}$ represents the $i$-th true tool wear value, and $y_{i}^{p}$ represents the $i$-th predicted tool wear value.

\begin{tabular}{|c|c|c|c|}
\hline Features & Expression & Features & Expression \\
\hline Mean value & $x_{\text {mean }}=\left(\sum_{i=1}^{N} x_{i}\right) / N$ & Minimum value & $x_{\min }=\min \left(x_{i}\right)$ \\
\hline Root mean square & $x_{r m s}=\sqrt{\left(\sum_{i=1}^{N} x_{i}^{2}\right) / N}$ & Peak-to-peak value & $x_{p t p}=x_{\max }-x_{\min }$ \\
\hline Square root value & $x_{s r a}=\left(\left(\sum_{i=1}^{N} \sqrt{\left|x_{i}\right|}\right) / N\right)^{2}$ & Standard deviation & $x_{s t d}=\sqrt{\left(\sum_{i=1}^{N}\left(\left|x_{i}\right|-\mu\right)^{2}\right) / N}$ \\
\hline Absolute mean & $x_{a m}=\left(\sum_{i=1}^{N}\left|x_{i}\right|\right) / N$ & Waveform index & $x_{\text {wave }}=x_{r m s} / x_{a m}$ \\
\hline Skewness & $x_{\text {ske }}=\left(\sum_{i=1}^{N}\left(\left|x_{i}\right|-x_{\text {mean }}\right)^{3} / x_{\text {std }}^{3}\right) / N$ & Peak index & $x_{p f}=x_{\max } / x_{r m s}$ \\
\hline Kurtosis & $x_{\text {ske }}=\left(\sum_{i=1}^{N}\left(\left|x_{i}\right|-x_{\text {mean }}\right)^{4} / x_{\text {std }}^{4}\right) / N$ & Impulse factor & $x_{i f}=x_{\max } / x_{a m}$ \\
\hline Maximum value & $x_{\min }=\max \left(x_{i}\right)$ & Tolerance index & $x_{t f}=x_{\max } / x_{s r a}$ \\
\hline \multicolumn{4}{|c|}{ Frequency domain } \\
\hline $\begin{array}{c}\text { Features } \\
\text { Spectrum centroid }\end{array}$ & $\begin{array}{c}\text { Expression } \\
x_{f c}=\sum_{n=1}^{N} f_{i} * P\left(f_{i}\right) / \sum_{i=1}^{N} P\left(f_{i}\right)\end{array}$ & $\begin{array}{c}\text { Features } \\
\text { Spectrum variance }\end{array}$ & $\begin{array}{c}\text { Expression } \\
x_{f v}=\sum_{n=1}^{N}\left(f_{i}-x_{f c}\right)^{2} * P\left(f_{i}\right) / \sum_{i=1}^{N} P\left(f_{i}\right)\end{array}$ \\
\hline
\end{tabular}

Table 1 List of extracted features

Time domain

Time-frequency domain 
where $\left\{x_{i}, i=1, \ldots, N\right\}$ denotes a signal sample. The time domain signal (i.e., the original signal) is transformed into power spectrum by fast Fourier transform and is expressed as $f_{i} . P\left(f_{i}\right)$ represents the power spectrum density, and $N$ represents the power spectrum length. For $r$-level wavelet packet decomposition, $M_{r, j}$ represents the ratio of the energy of the $j$-th sub band to the total energy of all sub bands. $k=1, \ldots, L / 2^{r}$ represents the wavelet packet coefficients, and $L$ represents the length of the original signal.

Table 2 Detailed structural parameters of the proposed ADSCNN model

\begin{tabular}{|c|c|c|c|c|c|}
\hline Layer name & Output shape & Kernel shape & Stride & Padding & $\begin{array}{l}\text { Activation } \\
\text { function }\end{array}$ \\
\hline Input layer & $48 \times 48 \times 3$ & - & - & - & - \\
\hline Position encoding layer & $48 \times 48 \times 12$ & - & - & - & - \\
\hline Self-attention layer & $48 \times 48 \times 12$ & - & - & - & - \\
\hline Concatenate layer & $96 \times 96 \times 3$ & - & - & - & - \\
\hline Convolution2D & $48 \times 48 \times 32$ & $3 \times 3 \times 3 \times 32$ & $2 \times 2$ & same & Relu \\
\hline Convolution2D dw & $48 \times 48 \times 32$ & $3 \times 3 \times 32$ & $1 \times 1$ & same & Relu \\
\hline Convolution2D & $48 \times 48 \times 64$ & $1 \times 1 \times 32 \times 64$ & $1 \times 1$ & same & Relu \\
\hline Convolution2D dw & $24 \times 24 \times 64$ & $3 \times 3 \times 64$ & $2 \times 2$ & same & Relu \\
\hline Convolution2D & $24 \times 24 \times 128$ & $1 \times 1 \times 64 \times 128$ & $1 \times 1$ & same & Relu \\
\hline Convolution2D dw & $24 \times 24 \times 128$ & $3 \times 3 \times 128$ & $1 \times 1$ & same & Relu \\
\hline Convolution2D & $24 \times 24 \times 128$ & $1 \times 1 \times 128 \times 128$ & $1 \times 1$ & same & Relu \\
\hline Convolution2D dw & $12 \times 12 \times 128$ & $3 \times 3 \times 128$ & $2 \times 2$ & same & Relu \\
\hline Convolution2D & $12 \times 12 \times 256$ & $1 \times 1 \times 128 \times 256$ & $1 \times 1$ & same & Relu \\
\hline Convolution2D dw & $12 \times 12 \times 256$ & $3 \times 3 \times 256$ & $1 \times 1$ & same & Relu \\
\hline Convolution2D & $12 \times 12 \times 256$ & $1 \times 1 \times 256 \times 256$ & $1 \times 1$ & same & Relu \\
\hline Convolution2D dw & $6 \times 6 \times 256$ & $3 \times 3 \times 256$ & $2 \times 2$ & same & Relu \\
\hline Convolution2D & $6 \times 6 \times 256$ & $1 \times 1 \times 256 \times 256$ & $1 \times 1$ & same & Relu \\
\hline GlobalAveragePooling2D & $1 \times 1 \times 256$ & $6 \times 6$ & - & - & Relu \\
\hline Flatten & 256 & - & - & - & - \\
\hline Dense & 128 & - & - & - & sigmoid \\
\hline Output & 3 & - & - & - & sigmoid \\
\hline
\end{tabular}

\section{Experimental data sets}

\subsection{Experimental setup}

A set of experimental data measured from three flute ball nose milling tool of computer numerical control machine in dry milling is used to verify the effectiveness of the proposed tool wear prediction method [36]. As shown in Fig. 4 , the CNC machine tool used is a high-speed CNC machine tool, the tool used is a ball nose milling cutter with three flutes, and the workpiece is an Inconel 718 alloy workpiece. Table 3 shows the process parameters of the cutting process. In the milling process, Kistler three-component dynamometer and Kistler three-component accelerometer are used to measure the milling force and vibration, respectively. A DAQ NI PCI1200 is used to collect the signals of multiple sensors, and the continuous sampling frequency is $50 \mathrm{kHz}$. The wear width of the flank of each cutting edge is measured with a LEICA MZ12 optical microscope after each milling.

Table 3 Process parameters of the experimental platform

\begin{tabular}{cc}
\hline Parameter & Value \\
\hline Spindle rotation speed & $173 \mathrm{r} / \mathrm{s}$ \\
X-axis feed rate & $26 \mathrm{~mm} / \mathrm{s}$ \\
y axis (radial) cutting depth & $125 \mu \mathrm{m}$ \\
z axis (axial) cutting depth & $200 \mu \mathrm{m}$ \\
Sampling frequency per channel & $50,000 \mathrm{HZ}$ \\
\hline
\end{tabular}

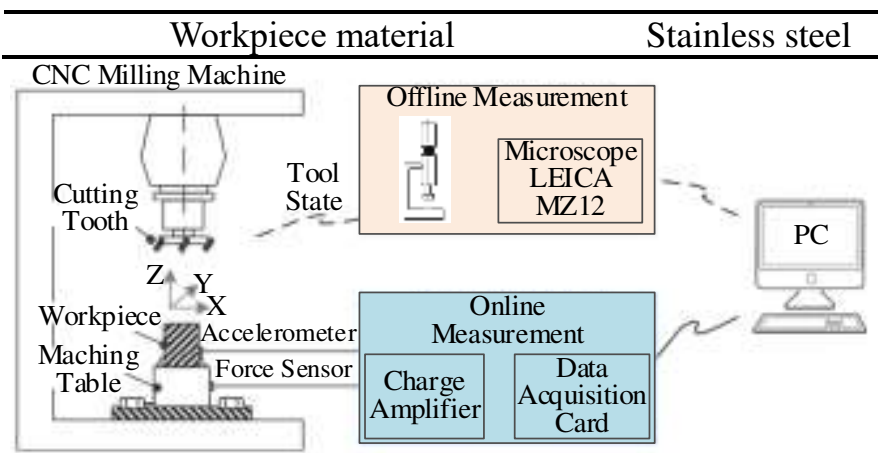

Fig. 4 The schematic diagram of the experimental platform

\subsection{Data preparation}

The tool wear data set consists of three tools $(C 1, C 4$ and $C 6)$ with complete tool wear data. Each tool contains 315 signal data files and corresponding tool wear data. Each signal data files corresponds to a cutting process. Here, the tool wear data set $C 1$ is taken as an example for data analysis. 


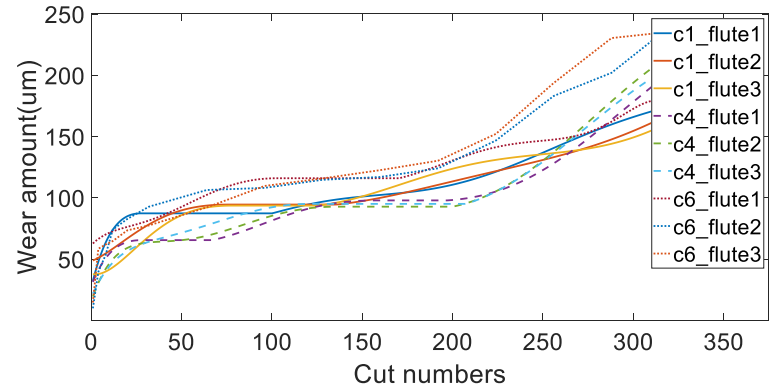

As shown in Fig. 5, the tool wears gradually with the increase in the number of cuts. For each tool, the change law of the wear curve of the three flutes is the same. Tool wear is divided into three stages: initial wear, normal wear and severe wear. In the initial wear stage, the slope of the curve is larger, and the tool wears faster. In the normal wear stage, the slope of the curve is small, and the tool wears slowly. In the severe wear stage, the slope of the curve is large, and the tool wears violently.

Fig. 5 Tool wear curve
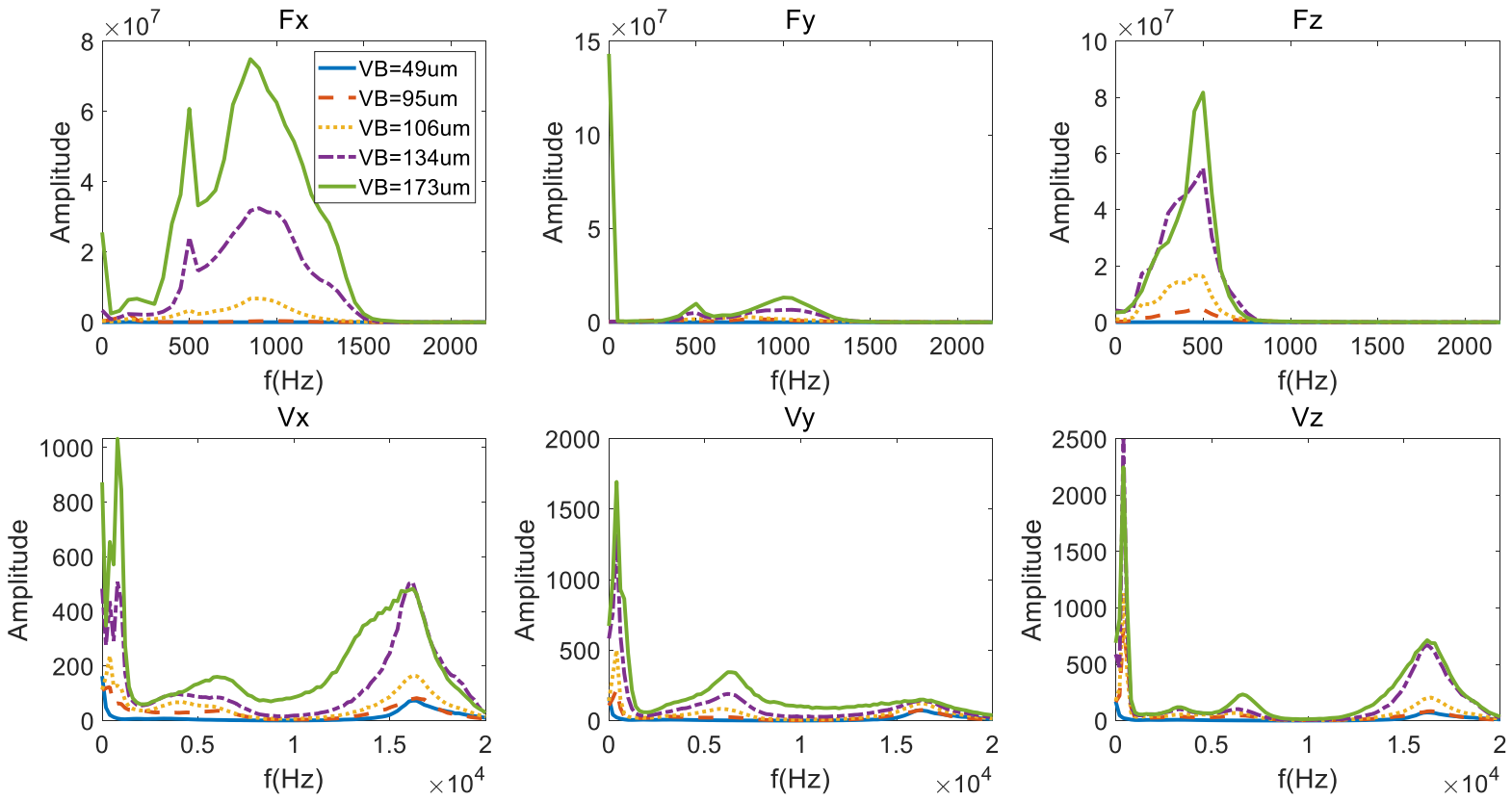

Fig. 6 Marginal spectra of milling force and vibration signals of $C 1$ under different tool wear status in three directions
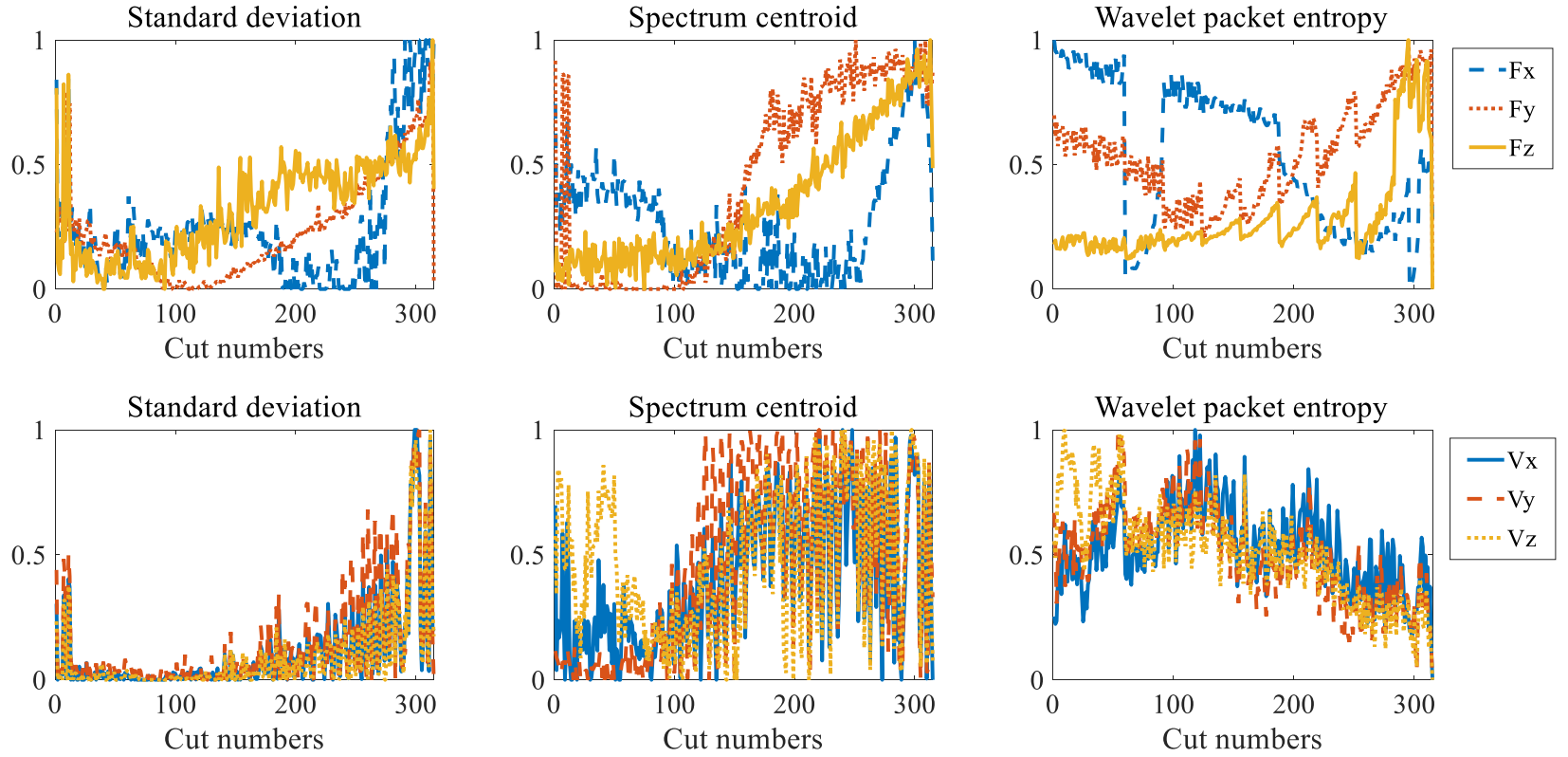

Fig. 7 Three normalized extracted features of milling force and vibration signals of $C 1$ in three directions

The marginal spectra of the milling force and vibration signals of $C 1$ under different tool wear status are shown in Fig. 6. The energy of the marginal spectrum of the first three milling force signals is mainly concentrated in the low frequency part $(0-1500 \mathrm{~Hz})$. The marginal spectra of the first three milling force signals are approximately unimodal. For the first three subgraphs, the amplitude of frequency distribution curve increases with the increase in tool wear. The amplitude of the frequency distribution curve of milling force in $\mathrm{x}$ direction and $\mathrm{z}$ direction is larger than that in $\mathrm{y}$ direction. The energy of the marginal spectrum of the last three vibration signals is dispersed over a wide frequency range $(0-20,000 \mathrm{~Hz})$. The marginal spectra of the last three vibration signals are approximately multimodal. For the last 
three subgraphs, the amplitude of frequency distribution curve increases with the increase in tool wear. The amplitude of the frequency distribution curve of $\mathrm{z}$-direction vibration is greater than that of $y$-direction and $\mathrm{x}$-direction vibrations. Therefore, the proposed tool wear monitoring strategy is effective in predicting tool wear in terms of cutting force and vibration signals in milling process.

Considering that the original milling force and vibration signals contain considerable noise, 24 multidomain features are extracted from each milling force and vibration signals. Three normalised extracted features of cutting force and vibration signals of $C 1$ are shown in Fig. 7. As shown in Fig. 7, some of the three features show a trend of decreasing and others increasing with the increase in cutting times. Therefore, predicting tool wear by using multidomain features is feasible. However, the relationship between tool wear and multidomain features is complex and nonlinear. Using the proposed ADSCNN model is necessary to model the nonlinear relationship between tool wear and multidomain features.

In accordance with the multidomain feature extraction method described in the previous section, the multidomain features of multiple sensor signals are extracted and reconstructed into multidomain feature tensors. The sample data sets composed of these multidomain feature tensors are divided into training data sets, validation data sets and test data sets. The detailed description of the experimental data sets is shown in Table 4. The detailed configuration of the computer used to train the ADSCNN model is as follows: Microsoft Windows 10 system, Intel (R) Core (TM) i5$3230 \mathrm{M}$ processor, 4 GB random access memory and $2 \mathrm{~Hz}$ $\mathrm{CPU}$ frequency. The training time of one epoch is approximately $2.14 \mathrm{~s}$, and the testing time of each sample is only $0.0015 \mathrm{~s}$. Therefore, the proposed ADSCNN model is an efficient tool wear prediction method.

Table 4 Description of experimental data sets

\begin{tabular}{lllll}
\hline $\begin{array}{l}\text { Tool } \\
\text { symbol }\end{array}$ & $\begin{array}{l}\text { Training } \\
\text { data sets }\end{array}$ & $\begin{array}{l}\text { Validation } \\
\text { data sets }\end{array}$ & $\begin{array}{l}\text { Test data } \\
\text { sets }\end{array}$ & $\begin{array}{l}\text { Total } \\
\text { data sets }\end{array}$ \\
\hline$C 1$ & 18900 & 6300 & 6300 & 31500 \\
$C 4$ & 18900 & 6300 & 6300 & 31500 \\
$C 6$ & 18900 & 6300 & 6300 & 31500 \\
\hline
\end{tabular}

\section{Results and discussion}

The performance of the proposed tool wear prediction method based on ADSCNN model depends on the selection of super parameter. The optimal super parameters of the ADSCNN model change with the experimental data sets. Therefore, in the application of the proposed method, optimising the super parameters of the ADSCNN model is necessary. Specifically, the parameters of the proposed ADSCNN model mainly include position encoding method, activation function of dense and output layer, gradient descent algorithm, learning rate and number of epochs. The early stop strategy avoids the selection of the number of epochs and can effectively avoid the occurrence of overfitting and improve the generalisation performance of the network. Therefore, the four parameters to be optimised include position encoding method, gradient descent algorithm, learning rate and activation function of dense and output layer. Considering the similarity of the wear process of three ball-end milling cutters, $C 1$ is used as a case of parameter selection.

\subsection{Position encoding algorithm}

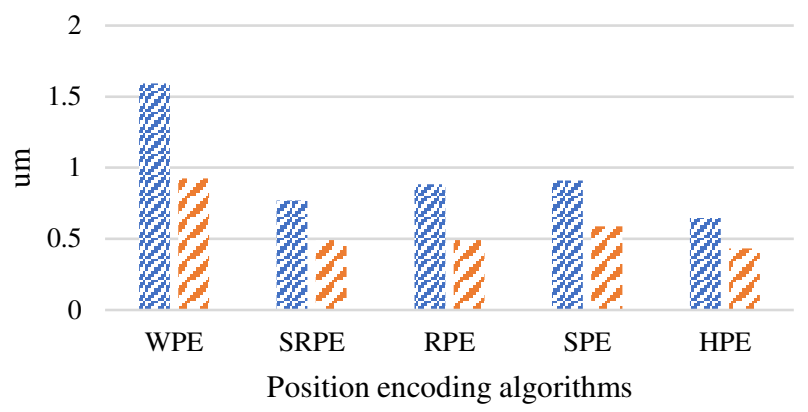

RMSE $>$ MAE

Fig. 8 Validation set accuracy of $C 1$ under different position encoding algorithms
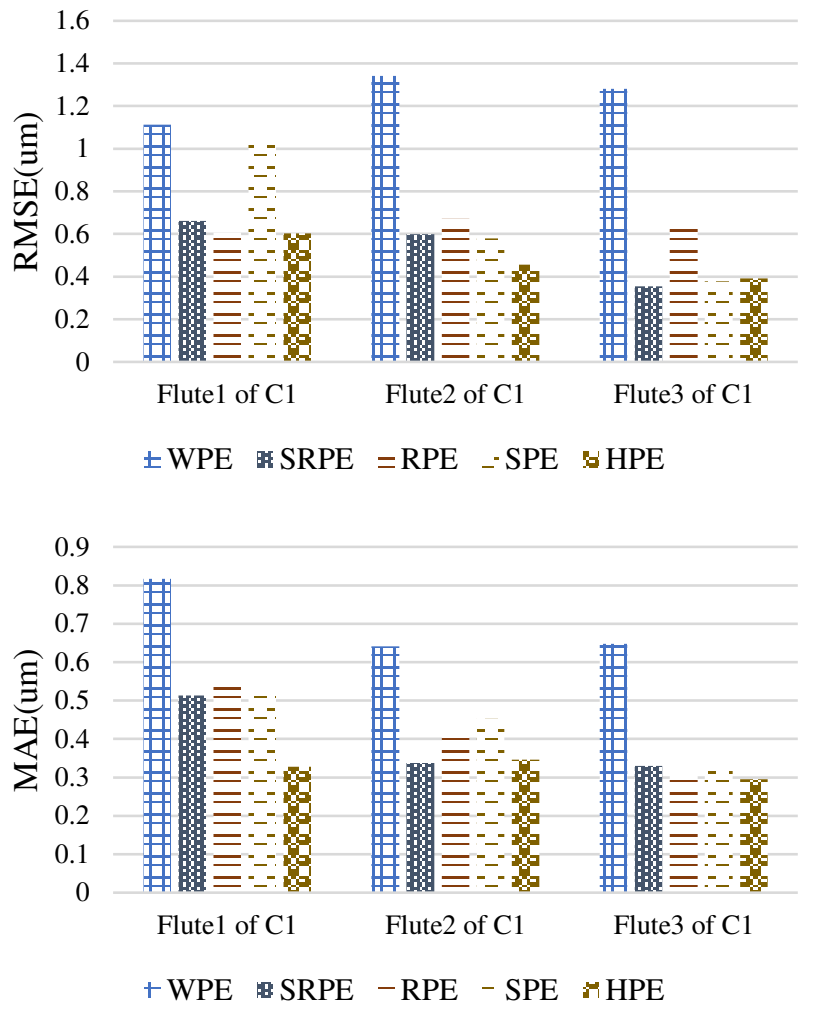

Fig. 9 Performance comparison of different flutes of $C 1$ under different position encoding algorithms The self-attention mechanism can't capture the position information between different elements in the input tensor, so it is often combined with the position encoding algorithm to enhance its performance. Therefore, the proposed hypercomplex position encoding algorithm is compared with several common position encoding algorithms (without position encoding, sinusoidal position encoding, relative position encoding, segmented relative position encoding and hypercomplex position encoding) to demonstrate its superiority. Hypercomplex position encoding algorithm is combined with high dimensional self-attention mechanism, and the other position encoding algorithms for comparison 
are combined with self-attention mechanism. Fig. 8 shows the validation accuracy under different position encoding algorithms. Fig. 9 compares the performance of different flutes of $C 1$ under different position encoding algorithms. As shown in Fig. 8, the ADSCNN model using position encoding algorithms reduces the MAE and RMSE by approximately $50 \%$ compared with the ADSCNN model without using position encoding technology. Sinusoidal position encoding is a static and no learnable encoding algorithm that encodes the position information of each feature vector but cannot represent the relative distance between feature vectors. Relative position encoding, segmented position encoding and hypercomplex position encoding are all learning position encoding algorithms. Relative position encoding only encodes the distance information between each pair of feature vectors that are close to each other. Segmented position encoding can encode the distance information between each pair of feature vectors at any distance, avoiding the distance limitation. As shown in Fig. 8, the ADSCNN model using hypercomplex position encoding has the lowest MAE and RMSE. As shown in Fig. 9, the ADSCNN model using hypercomplex position encoding has the lowest RMSE and MAE for different flutes of $C 1$. Hypercomplex position encoding regards the encoded vector corresponding to each feature vector as a continuous function of position, thereby avoiding the distance limitation. Therefore, the proposed hypercomplex position encoding and the corresponding high dimensional self-attention mechanism are embedded in the proposed ADSCNN model to enhance the useful information and suppress the noise in the input tensor.

\subsection{Activation function}
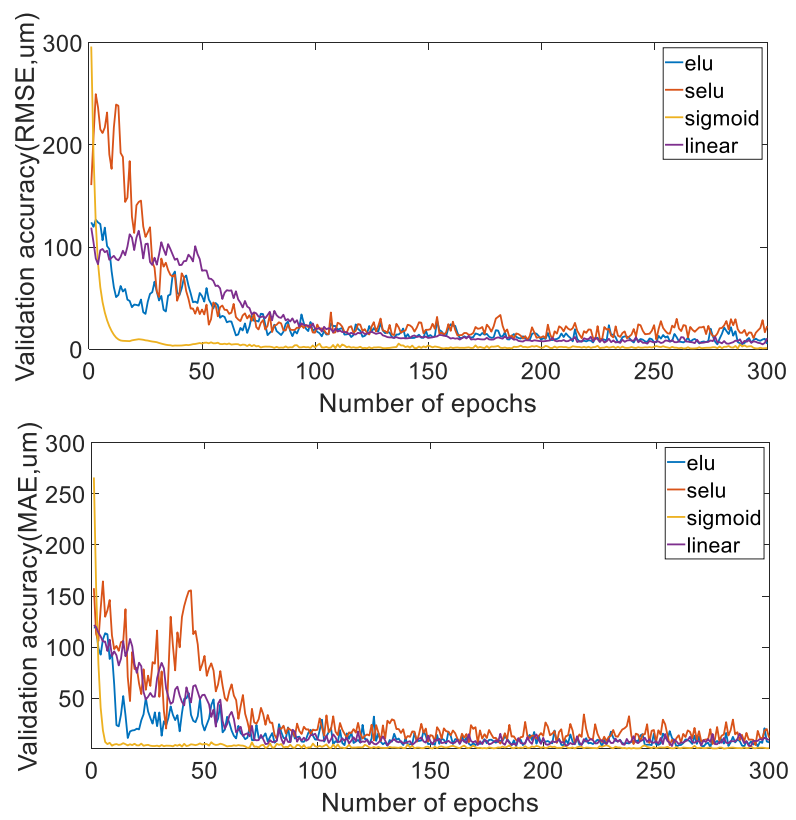

Fig. 10 Validation set accuracy of $C 1$ under different activation functions
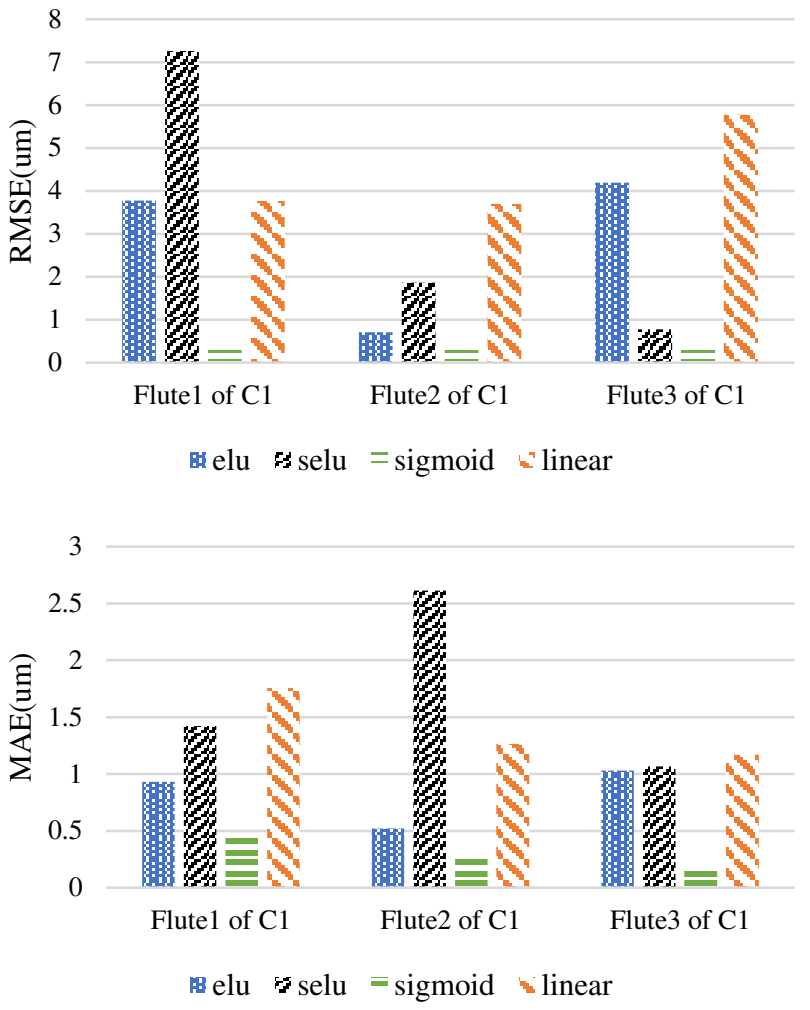

Fig. 11 Performance comparison of different flutes of $C 1$ under different activation functions

The activation function directly affects the capacity and parameter optimisation of neural network model. At present, a variety of activation functions have been widely used. However, no complete theoretical guidance is reported on how to select the appropriate activation function for specific applications. Therefore, common activation functions (including elu, selu, sigmoid and linear) are compared to choose a suitable activation function. Fig. 10 shows the change trend of validation set accuracy under different activation functions. Fig. 11 shows the performance of different flutes of $C 1$ under different activation functions. As shown in Fig. 10, the ADSCNN model using sigmoid activation function converges faster and can converge to smaller MAE and RMSE compared with three linear activation functions (including linear, elu, and selu). The three linear activation functions are extremely fragile in the training process, easily leading to neuronal necrosis. The three activation functions do not compress the output amplitude, leading to the continuous expansion of output amplitude. The input of the proposed ADSCNN model is normalised multidomain features, and its expected output should be in the interval $[0,1]$. Fortunately, the sigmoid activation function can control the output in the interval $[0,1]$ without causing gradient explosion. As shown in Fig. 11, the ADSCNN model using sigmoid activation function has the lowest RMSE and MAE for different flutes of $C 1$. Therefore, the sigmoid activation function is used as the activation function of the dense and output layer.

\subsection{Gradient descent algorithm}



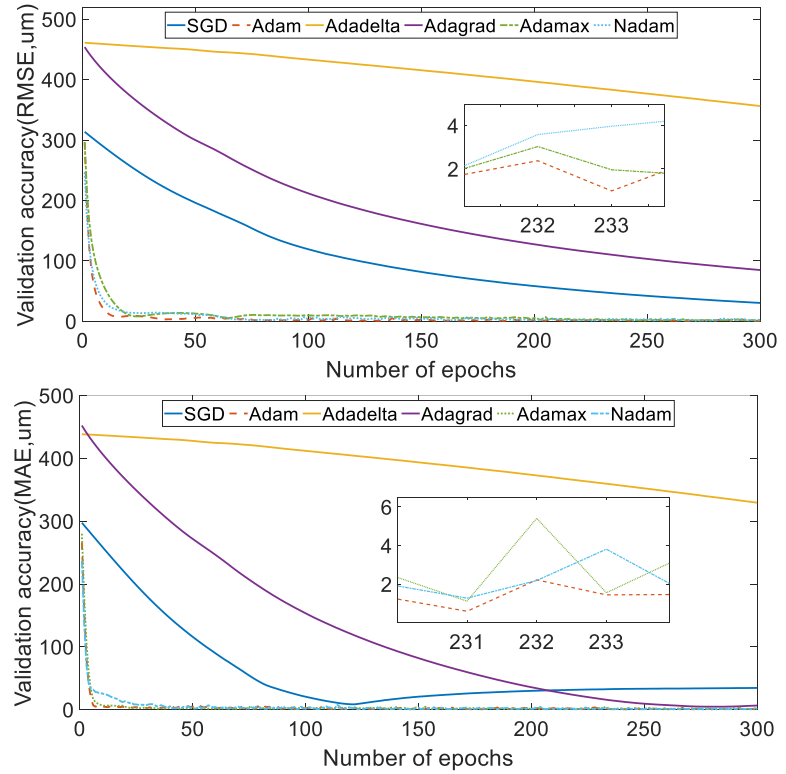

Fig. 12 Validation set accuracy of $C 1$ under different gradient descent algorithm

1000

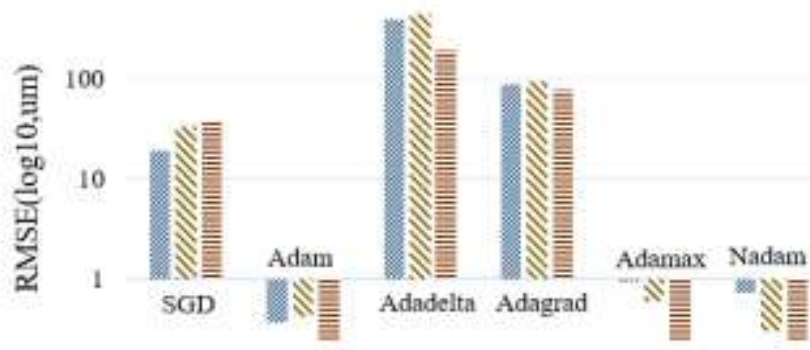

0.1

= flutel of $\mathrm{Cl} \approx$ f flute 2 of $\mathrm{Cl} \equiv$ flute 3 of $\mathrm{Cl}$

1000

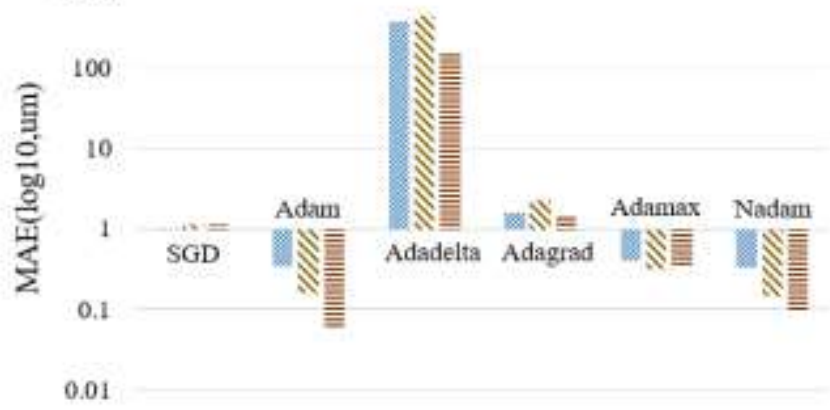

w flutel of $\mathrm{Cl}$ : flute2 of $\mathrm{Cl}=$ flute 3 of $\mathrm{Cl}$

Fig. 13 Performance comparison of different flutes of $C 1$ under different gradient descent algorithm

As a popular optimisation algorithm, gradient descent algorithm is often used to optimise neural network models. Different optimisation algorithms are often regarded as black box optimisers due to the lack of theoretical explanation for their advantages and disadvantages. Therefore, common optimisation algorithms (including stochastic gradient descent [SGD], adaptive moment estimation [Adam], Adadelata, Adagrad, Adamax and Nadam) are compared to choose a suitable optimisation algorithm. The change trend of validation set accuracy of $C 1$ under different gradient descent algorithms is shown in Fig. 12. As shown in Fig. 13, the performance of different flutes of $C 1$ under different gradient descent algorithms is evaluated. As shown in Fig. 12, SGD, Adadelata and Adagrad have a slow convergence rate and a large validation error. Adam, Adamax and Nadam all have fast convergence speeds. As shown in Fig. 13, Adam achieves the smallest MAE and RMSE compared with Adamax and Nadam. Therefore, Adam is chosen to train the proposed ADSCNN model.

\subsection{Learning rate}
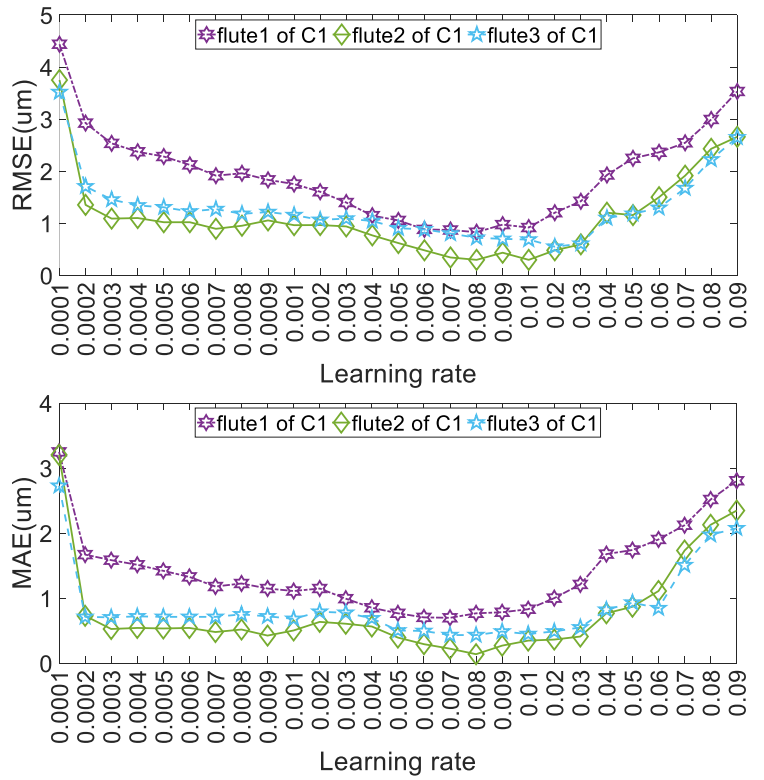

Fig. 14 Performance comparison of different flutes of $C 1$ under different learning rates

In the model training, gradient descent algorithm is often used to optimise the super parameters of the model. For the gradient descent algorithm, the learning rate is an important parameter that directly affects the convergence speed of the model and its convergence error. Choosing the appropriate learning rate is necessary to improve the efficiency and accuracy of the proposed ADSCNN model. Therefore, different learning rates are used to train the proposed ADSCNN model. The results of performance metrics (including MAE and RMSE) under different learning rates are shown in Fig. 14. With the increase in learning rate, RMSE and MAE decrease first and then increase. Specifically, RMSE and MAE are the smallest when the learning rate is approximately 0.008 . The smaller the RMSE and MAE, the closer the predicted tool wear value is to the actual flank wear value. The appropriate learning rate can reduce the prediction error and accelerate the model convergence. Therefore, considering the prediction accuracy and convergence speed, the appropriate learning rate is set to 0.008 .

\subsection{Comparison of different tool wear prediction methods}

Table 5 The optimal parameters of the proposed tool wear prediction method

Parameters Gradient descent algorithm Learning rate
Position encoding algorithm

Activation function 

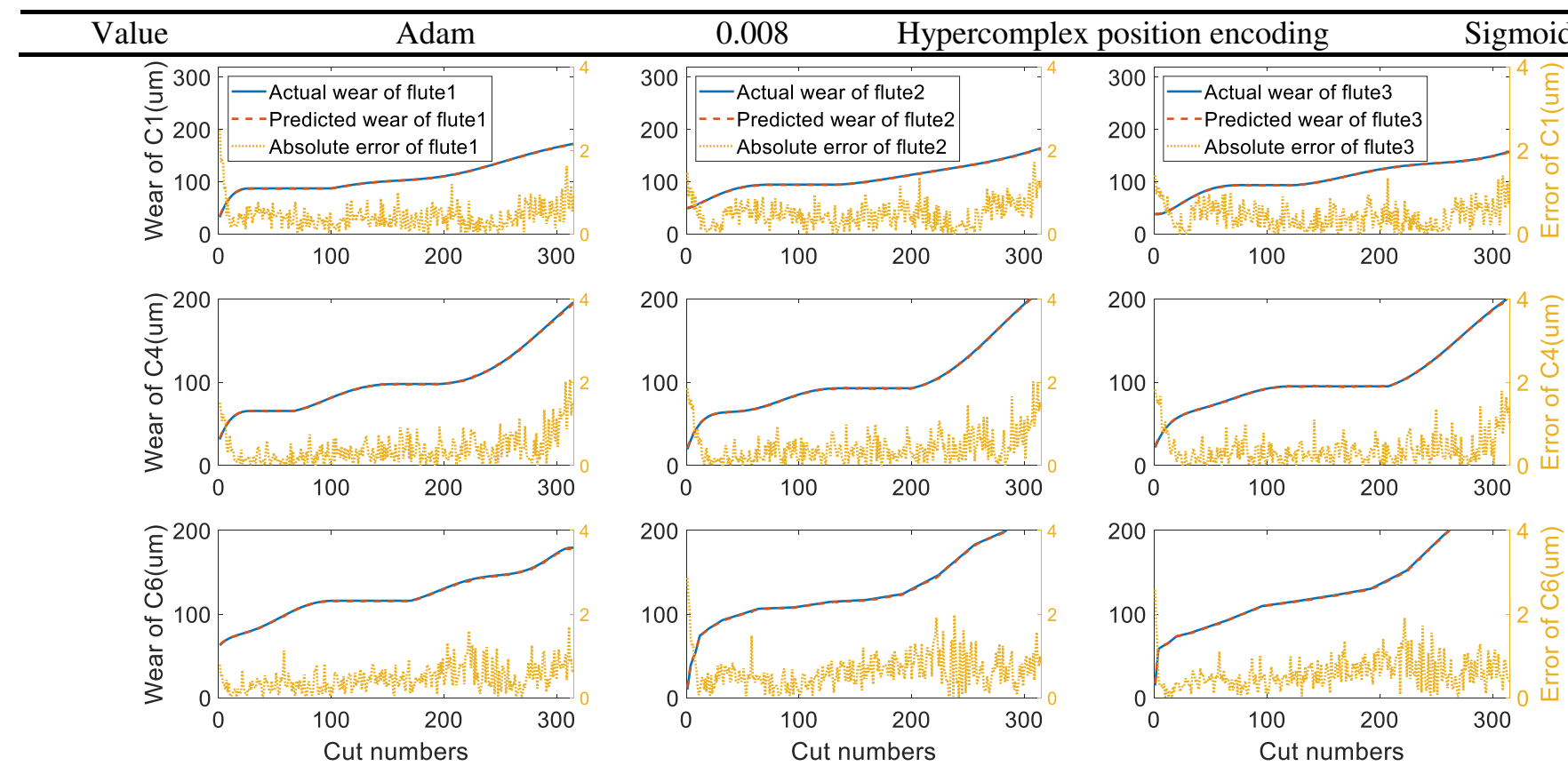

Fig. 15 The prediction results of the proposed tool wear prediction method

Table 6 Performance comparison of different tool wear prediction methods

\begin{tabular}{ccc}
\hline Method & $\operatorname{RMSE}(\mu \mathrm{m})$ & $\operatorname{MAE}(\mu \mathrm{m})$ \\
\hline SVR[5] & $11.9681 \pm 3.3337$ & $9.3770 \pm 2.0422$ \\
SVR+KPCA[5] & $5.4428 \pm 1.5894$ & $3.9583 \pm 0.9371$ \\
CNN[37] & $14.0428 \pm 5.5588$ & $11.0000 \pm 1.3000$ \\
DCNN+AE[38] & $2.2385 \pm 0.7105$ & $1.5708 \pm 0.5485$ \\
CBLSTM[12] & $9.2333 \pm 1.9140$ & $7.2333 \pm 1.0263$ \\
LSTM[12] & $13.7333 \pm 6.2164$ & $12.1667 \pm 6.2292$ \\
RNN[12] & $15.7333 \pm 6.2164$ & $12.1667 \pm 6.2292$ \\
RSTCNN[15] & $2.2009 \pm 0.9012$ & $1.5658 \pm 0.6037$ \\
ADSCNN & $\mathbf{0 . 6 8 7 8} \pm \mathbf{0 . 3 8 3 1}$ & $\mathbf{0 . 4 7 3 8} \pm \mathbf{0 . 1 8 2 2}$ \\
\hline
\end{tabular}

In accordance with a series of parameter optimisation experiments, the optimal parameters of the proposed ADSCNN model are shown in Table 5. The tool wear prediction values and prediction errors for data sets $C 1, C 4$ and $C 6$ are shown in Fig. 15. The tool wear prediction error for each cutting number is less than $5 \mu \mathrm{m}$. The proposed tool wear prediction method can accurately predict tool flank wear.

The performance of the proposed method is compared with other advanced methods by using original published data to demonstrate its effectiveness and advancement. Specifically, traditional machine learning methods, including SVR+KPCA and SVR, and advanced deep learning methods, including recurrent neural network (RNN), LSTM, CNN, CBLSTM, DCNN+AE and RTSCNN, are used for performance comparison. The comparison results under different evaluation criteria are shown in Table 6. SVR is unsuitable for large-scale data sets, difficult to select the kernel function, and has high prediction error. KPCA improves the prediction accuracy of SVR by fusing multidomain features, but the prediction accuracy is still lower than the proposed tool wear prediction method. RNN, LSTM, CBLSTM, CNN and DCNN+AE directly model the relationship between multidomain features and tool flank wear, but their prediction error is higher than that of the proposed tool wear prediction method. Compared with other comparative methods, the MAE and RMSE of the proposed tool wear prediction method are the smallest, which are 0.47 and $0.69 \mu \mathrm{m}$, respectively. The variances of MAE and RMSE are the smallest, which are 0.18 and $0.38 \mu \mathrm{m}$, respectively. The proposed tool wear prediction method has the lowest variance and deviation, and has best prediction performance. In conclusion, the proposed tool wear prediction method can accurately and effectively model the nonlinear relationship between tool wear and multidomain features and achieve high prediction accuracy.

\section{Conclusion}

In this paper, a new tool wear prediction method based on multidomain feature fusion by ADSCNN for milling process is proposed. This method improves the accuracy of tool wear prediction. The proposed method can achieve good prediction accuracy mainly due to the following technologies. First, the multisensory signal contains considerable tool wear information. Multidomain feature extraction provides comprehensive and rich features to characterise tool wear. Second, proposed hypercomplex position encoding and high dimensional self-attention mechanism are used to calculate the new representation of input feature tensor, which emphasizes the tool wear sensitive information and suppresses large area background noise. Third, the proposed depth-wise separable CNN is used to model the nonlinear relationship between tool wear and the new representation. Fourth, the proposed ADSCNN model adopts a dense layer and an output layer to automatically predict tool wear. Finally, on the basis of a variety of metrics, the parameters of the proposed ADSCNN model are optimised, thereby helping to further improve its prediction accuracy.

The main contribution of this paper is to realise the automatic prediction of tool wear in milling process by using the ADSCNN model to adaptively fuse multidomain features and omit the steps of feature selection and feature 
dimension reduction on the basis of feature engineering technology. In addition, the innovation is that the proposed hypercomplex position encoding and high dimensional selfattention mechanism enhances the tool wear sensitive information contained in the input feature tensor and suppresses the noise, and the proposed depth-wise separable CNN can well model the nonlinear relationship between tool wear and the new representation. The experimental results of three milling tool run-to-failure data sets show that the proposed tool wear prediction method based on ADSCNN model is better than other state-of-art methods in terms of overall prediction performance. The proposed tool wear prediction method based on ADSCNN model shows good prediction performance in the milling process. The findings serve as reference for its application in machining.

Author contribution Yanbo Wang conceived of the presented idea and developed the theoretical formalism. Yongchao Huo aided in interpreting the results. All authors discussed the results and contributed to the final manuscript.

Funding This work is supported by National Natural Science Foundation of China (51905209), Natural Science Foundation of Jilin Province (2020122332JC).

Data availability The data that support the findings of this study are available from the website, https://phmsociety.org/phm competition/2010-phm-

society-conference-data-challenge/.

Code availability The original code of this study is available from corresponding author, Yanbo Wang, upon reasonable request.

\section{Declarations}

Ethical approval Not applicable

Consent to participate Not applicable

Consent for publication Not applicable

Competing interests The authors declare no competing interests.

\section{References}

1. Zhu KP, Zhang Y (2019) A generic tool wear model and its application to force modeling and wear monitoring in high speed milling. Mech Syst Signal Proc https://doi:10.1016/j.ymssp.2018.05.045

2. Chi YJ, Dai W, Lu ZY, Wang MQ, Zhao Y (2018) RealTime Estimation for Cutting Tool Wear Based on Modal Analysis of Monitored Signals. Appl Sci-Basel 8 (5):13. https://doi:10.3390/app8050708
3. Morgan J, O'Donnell GE (2018) Cyber physical process monitoring systems. J Intell Manuf 29 (6):1317-1328. https://doi:10.1007/s10845-015-1180-Z

4. Zhang KF, Yuan HQ, Nie P (2015) A method for tool condition monitoring based on sensor fusion. J Intell Manuf 26 (5):1011-1026. https://doi:10.1007/s10845015-1112-y

5. Wang JJ, Xie JY, Zhao R, Zhang LB, Duan LX (2017) Multisensory fusion based virtual tool wear sensing for ubiquitous manufacturing. Robot Comput-Integr Manuf 45:47-58. https://doi:10.1016/j.rcim.2016.05.010

6. Pandiyan V, Caesarendra W, Tjahjowidodo T, Tan $\mathrm{HH}$ (2018) In-process tool condition monitoring in compliant abrasive belt grinding process using support vector machine and genetic algorithm. J Manuf Process 31:199-213. https://doi:10.1016/j.jmapro.2017.11.014

7. Kong DD, Chen YJ, Li N (2018) Gaussian process regression for tool wear prediction. Mech Syst Signal Proc 104:556-574. https://doi:10.1016/j.ymssp.2017.11.021

8. Li G, Wang Y, He J, Hao Q, Yang H, Wei J (2020) Tool wear state recognition based on gradient boosting decision tree and hybrid classification RBM. International Journal of Advanced Manufacturing Technology $110 \quad$ (1-2):511-522. https://doi:10.1007/s00170-020-05890-x

9. Wu J, Su YH, Cheng YW, Shao XY, Deng C, Liu C (2018) Multi-sensor information fusion for remaining useful life prediction of machining tools by adaptive network based fuzzy inference system. Appl Soft Comput 68:13-23. https://doi:10.1016/j.asoc.2018.03.043

10. Fu Y, Zhang Y, Gao Y, Gao H, Mao T, Zhou HM, Li DQ (2017) Machining vibration states monitoring based on image representation using convolutional neural networks. Eng Appl Artif Intell 65:240-251. https://doi:10.1016/j.engappai.2017.07.024

11. Aghazadeh F, Tahan A, Thomas M (2018) Tool condition monitoring using spectral subtraction and convolutional neural networks in milling process. International Journal of Advanced Manufacturing Technology $98 \quad$ (9-12):3217-3227. https://doi:10.1007/s00170-018-2420-0

12. Zhao R, Yan RQ, Wang JJ, Mao KZ (2017) Learning to Monitor Machine Health with Convolutional BiDirectional LSTM Networks. Sensors 17 (2):18. https://doi:10.3390/s17020273

13. Cheng C, Li JY, Liu YM, Nie M, Wang WX (2019) Deep convolutional neural network-based in-process tool condition monitoring in abrasive belt grinding. Comput Ind 106:1-13. https://doi:10.1016/j.compind.2018.12.002

14. Chen, Xie, Yuan, Huang, Li (2019) Research on a Real-Time Monitoring Method for the Wear State of a Tool Based on a Convolutional Bidirectional LSTM Model. Symmetry 11 (10):1233

15. Huang ZW, Zhu JM, Lei JT, Li XR, Tian FQ (2019) Tool Wear Predicting Based on Multisensory Raw Signals Fusion by Reshaped Time Series Convolutional 
Neural Network in Manufacturing. IEEE Access 7:178640-178651.

https://doi:10.1109/access.2019.2958330

16. Martinez-Arellano G, Terrazas G, Ratchev S (2019) Tool wear classification using time series imaging and deep learning. International Journal of Advanced Manufacturing Technology 104 (9-12):3647-3662. https://doi:10.1007/s00170-019-04090-6

17. Cao XC, Chen BQ, Yao B, He WP (2019) Combining translation-invariant wavelet frames and convolutional neural network for intelligent tool wear state identification. Comput Ind 106:71-84. https://doi:10.1016/j.compind.2018.12.018

18. Song KY, Wang M, Liu LM, Wang C, Zan T, Yang B (2020) Intelligent recognition of milling cutter wear state with cutting parameter independence based on deep learning of spindle current clutter signal. International Journal of Advanced Manufacturing Technology $109 \quad$ (3-4):929-942. https://doi:10.1007/s00170-020-05587-1

19. Sun HB, Zhang JD, Mo R, Zhang XZ (2020) Inprocess tool condition forecasting based on a deep learning method. Robot Comput-Integr Manuf 64:8. https://doi:10.1016/j.rcim.2019.101924

20. Li YT, Xie QS, Huang HS, Chen QP (2019) Research on a Tool Wear Monitoring Algorithm Based on Residual Dense Network. Symmetry-Basel 11 (6):19. https://doi:10.3390/sym11060809

21. Shang RH, He JH, Wang JM, Xu KM, Jiao LC, Stolkin $\mathrm{R}$ (2020) Dense connection and depthwise separable convolution based CNN for polarimetric SAR image classification. Knowledge-Based Syst 194:13. https://doi:10.1016/j.knosys.2020.105542

22. Huang GJ, Zhang YL, Ou JY (2021) Transfer remaining useful life estimation of bearing using depthwise separable convolution recurrent network. Measurement 176:13. https://doi:10.1016/j.measurement.2021.109090

23. Chollet F, Ieee (2017) Xception: Deep Learning with Depthwise Separable Convolutions. In: 30th Ieee Conference on Computer Vision and Pattern Recognition. IEEE Conference on Computer Vision and Pattern Recognition. Ieee, New York, pp 1800-1807. doi:10.1109/cvpr.2017.195

24. Xin B, Zheng YL, Wang TZ, Chen LS, Wang YD A diagnosis method based on depthwise separable convolutional neural network for the attachment on the blade of marine current turbine. Proc Inst Mech Eng Part I-J Syst Control Eng:11. https://doi:10.1177/0959651820937841

25. Howard AG, Zhu M, Chen B, Kalenichenko D, Wang W, Weyand T, Andreetto M, Adam H (2017) MobileNets: Efficient Convolutional Neural Networks for Mobile Vision Applications.

26. Han H, Wang H, Liu Z, Wang J (2021) Intelligent vibration signal denoising method based on non-local fully convolutional neural network for rolling bearings. ISA transactions.
27. Wang X, Girshick R, Gupta A, He K (2017) Non-local Neural Networks. Paper presented at the 2018 IEEE Conference on Computer Vision and Pattern Recognition, Salt Lake City,USA.,

28. Shokri M, Harati A, Taba K (2020) Salient object detection in video using deep non-local neural networks. $\mathrm{J}$ Vis Commun Image Represent 68:10. https://doi:10.1016/j.jvcir.2020.102769

29. Zeng YF, Liu RL, Liu XF (2021) A novel approach to tool condition monitoring based on multi-sensor data fusion imaging and an attention mechanism. Meas Sci Technol 32 (5):17. https://doi:10.1088/13616501/abea3f

30. Wang BA, Lei YG, Li NP, Wang WT (2021) Multiscale Convolutional Attention Network for Predicting Remaining Useful Life of Machinery. IEEE $\begin{array}{llll}\text { Trans Ind Electron } 68 \text { (8):7496-7504. } & \text {. }\end{array}$ https://doi:10.1109/tie.2020.3003649

31. Huang YR, Chen JJ, Zheng SM, Xue Y, Hu XH (2021) Hierarchical multi-attention networks for document classification. Int J Mach Learn Cybern 12 (6):16391647. https://doi:10.1007/s13042-020-01260-x

32. Vaswani A, Shazeer NM, Parmar N, Uszkoreit J, Jones L, Gomez AN, Kaiser L, Polosukhin I (2017) Attention is All you Need. Advances in Neural Information Processing Systems

33. Liu H, Liu ZY, Jia WQ, Lin XK, Zhang S (2020) A novel transformer-based neural network model for tool wear estimation. Meas Sci Technol 31 (6):12. https://doi:10.1088/1361-6501/ab7282

34. Shaw P, Uszkoreit J, Vaswani A Self-Attention with Relative Position Representations. In: Proceedings of the 2018 Conference of the North American Chapter of the Association for Computational Linguistics: Human Language Technologies, Volume 2 (Short Papers), 2018.

35. Dai Z, Yang Z, Yang Y, Carbonell J, Salakhutdinov R Transformer-XL: Attentive Language Models beyond a Fixed-Length Context. In: Proceedings of the 57th Annual Meeting of the Association for Computational Linguistics, 2019.

36. Li X, Lim BS, Zhou J, Huang S, Phua SJ, Shaw KC, Er MH Fuzzy Neural Network Modelling for Tool Wear Estimation in Dry Milling Operation. In, 2009.

37. Zhao R, Yan RQ, Chen ZH, Mao KZ, Wang P, Gao RX (2019) Deep learning and its applications to machine health monitoring. Mech Syst Signal Proc 115:213-237. https://doi:10.1016/j.ymssp.2018.05.050

38. Zhang C, Yao X, Zhang J, Liu E (2017) Tool wear monitoring based on deep learning. Jisuanji Jicheng Zhizao Xitong/Computer Integrated Manufacturing Systems, CIMS 23 (10):2146-2155. https://doi:10.13196/j.cims.2017.10.008 